\title{
Effect of External Cuff Pressure on Arterial Compliance
}

\author{
D Zheng, J Allen, A Murray \\ Medical Physics Department, University of Newcastle upon Tyne, Freeman Hospital, \\ Newcastle upon Tyne, UK
}

\begin{abstract}
Pressure is one of the most sensitive factors influencing the arterial wall compliance. Pulse transit time (PTT) is one simple way to assess arterial compliance changes. This study investigated the relationship between external cuff pressure and pulse transit time. The study was performed on 10 subjects with different cuff pressures (0, 10, 20 and $30 \mathrm{mmHg}$ ) on the lower arm, upper arm and whole arm (with two cuffs applied simultaneously on the lower arm and upper arm). The overall mean pulse transit time difference with the above different cuff pressures on the lower arm compared with that without cuff pressure were $3.3 \mathrm{~ms}, 6.9 \mathrm{~ms}, 10.1$ ms. With the cuff on the upper arm, changes with increasing cuff pressure were greater, which were $3.7 \mathrm{~ms}$, $8.5 \mathrm{~ms}$ and $13.1 \mathrm{~ms}$. And for the whole arm, the changes were $5.6 \mathrm{~ms}$, $11.6 \mathrm{~ms}$ and $18.0 \mathrm{~ms}$, which were greater still. The mean PTT when the cuff pressure was applied to the whole arm was significantly larger $(P<0.001)$ than the corresponding mean PTT when the cuff pressure was separately applied to the lower arm and upper arm. The mean PTT with the cuff on upper arm was also significantly larger than the corresponding mean PTT with the cuff on the lower arm, except with a cuff pressure of $10 \mathrm{mmHg}$. The sum of PTT changes with the cuff on the upper and the lower arm was significantly larger than that with the cuff on whole arm. A conclusion was made that when the external cuff pressure increased, arterial wall compliance increased, which was detected with increased pulse transit time.
\end{abstract}

\section{Introduction}

The compliance of the arterial system plays an important role in cardiovascular physiology and pathophysiology. Currently, there are three main noninvasive methodologies for assessing arterial compliance, which are based on analysis of pulse wave velocity [1], of pulse wave contour [2], and of direct measurement of arterial geometry and pressure [3]. It has also been known that a lot of factors influence arterial wall compliance, such as arterial pressure, temperature and age [4-5]. Among them, pressure is one of the most sensitive factors
[6-8], which is the difference between the internal pressure $(\mathrm{Pi})$ and external pressure $(\mathrm{Po})$, called transmural pressure. Internal pressure is the same as the blood pressure.

Pulse transit time (PTT), which is the time taken for the pulse pressure wave to travel from the heart to the peripheral artery, has been widely used for assessing arterial compliance changes. In this study, the technique based on photoplethysmography was used to obtain the pulse transit time, which is simple, non-invasive, quick and sensitive.

The objective of this study was to investigate how the external cuff pressure affects arterial compliance and pulse transit time.

\section{Methods}

\subsection{Subjects}

Ten healthy volunteers with no history of cardiovascular disease were studied. There were 5 male and 5 female subjects, with ages in the range of 21 to 32 years. All subjects were asked for consent.

\subsection{Measurement system}

Figure 1 shows the schematic representation of the measurement system. In the system, the cuff (Accoson Blood pressure cuff standard adult 228 x 127mm) was applied to the lower arm, upper arm and whole arm (with two small cuffs applied simultaneously on the lower arm and upper arm) to cause the changes of external pressure. One reflectance mode PPG probe (Artema, Denmark: type 75333-5) was used to pick up the changes in infrared reflectance resulting from varying blood volume. The probe was placed flat in the centre of the finger pulp of the index finger, which was attached using black Velcro cuffs to reduce ambient artefacts. A three-electrode ECG signal was also recorded to provide a timing reference for the PPG pulses. The signals from PPG probe and ECG electrodes were then fed separately to their own amplifier and filter. A data capture computer with sampling rate of $2500 \mathrm{~Hz}$ was used to convert the analog physiological signals to digital signals for subsequent off-line analysis. 


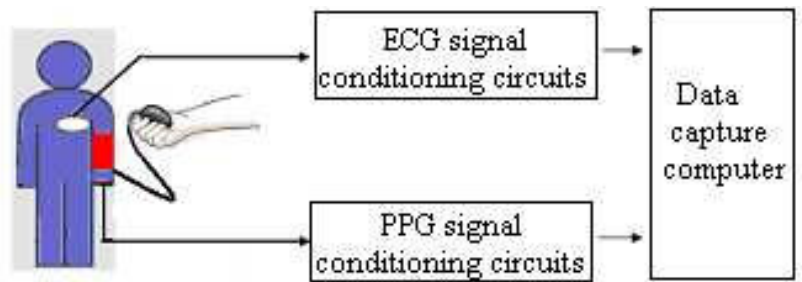

Figure 1: Measurement system

\subsection{Measurement protocol}

All the measurements were undertaken in a temperature-controlled room $\left(23 \pm 1^{\circ} \mathrm{C}\right)$ at the Medical Physics department of Freeman Hospital. The room was kept quiet during measurement.

Before the experiment, the subject was asked not to drink coffee for an hour and not to undergo strenuous exercise. The subject was required to lie supine on the measurement couch and then to relax for five minutes. During the whole measurement session, the subject was required to breathe steadily. The hands were kept at heart level covered with a towel. And the subject was also asked to remain still to reduce motion artefact.

All the measurements were done by the same operator to ensure the probes and the cuffs were attached with similar tightness. The recording time was 120 s for each test.

\subsection{Data collection}

For each subject, a series of 48 separate tests were performed with the different cuff pressures $(0,10,20$ and $30 \mathrm{mmHg}$ ) at different arm positions based on a randomized experiment design, as shown in the table 1. Each cuff pressure was applied four times, but in a different order. With the same randomized design, the procedure was repeated on their upper arm for tests 1732. For tests 33-48, two cuffs were used together, one on the upper arm and the other one on the lower arm. After each measurement, the cuff pressure was released, and the gap between each test was 30s.

\subsection{Pulse wave analysis and data statistics}

Pulses were first pre-processed by filtering to removing the higher frequency noise components. After filtering stage, if the signals were still very poor because of motion artifacts, these beats were excluded. The pulse transit time was calculated off-line from the ECG Q wave to the corresponding foot of PPG in each beat using MATLAB 6.1 analysis software. It is understood that the pulse transit time includes the pre-ejection time, but since this study examined PTT changes, this was still acceptable without extracting the pre-ejection time.

\begin{tabular}{|c|c|c|c|c|}
\hline Test & $\begin{array}{c}\text { Cuff } \\
\text { position }\end{array}$ & $\begin{array}{c}\text { Cuff } \\
\text { Pressure } \\
(\mathrm{mmHg})\end{array}$ & $\begin{array}{c}\text { Signal } \\
\text { recording } \\
\text { time }(\mathrm{s})\end{array}$ & $\begin{array}{c}\text { Recovery } \\
\text { time(s) } \\
\text { (Release } \\
\text { pressure) }\end{array}$ \\
\hline 1 & Lower & 0 & 120 & 30 \\
\hline 2 & Lower & 10 & 120 & 30 \\
\hline 3 & Lower & 20 & 120 & 30 \\
\hline 4 & Lower & 30 & 120 & 30 \\
\hline 5 & Lower & 10 & 120 & 30 \\
\hline 6 & Lower & 0 & 120 & 30 \\
\hline 7 & Lower & 30 & 120 & 30 \\
\hline 8 & Lower & 20 & 120 & 30 \\
\hline 9 & Lower & 20 & 120 & 30 \\
\hline 10 & Lower & 30 & 120 & 30 \\
\hline 11 & Lower & 10 & 120 & 30 \\
\hline 12 & Lower & 0 & 120 & 30 \\
\hline 13 & Lower & 30 & 120 & 30 \\
\hline 14 & Lower & 20 & 120 & 30 \\
\hline 15 & Lower & 0 & 120 & 30 \\
\hline 16 & Lower & 10 & 120 & 30 \\
\hline
\end{tabular}

Table 1: Cuff pressure and position information for tests $1-16$

The median of pulse transit time from 60 consecutive beats for each different cuff pressure on different positions was calculated individually for each subject. The pulse transit time difference when the cuff was applied to different positions under the same cuff pressure was also calculated and summarized. Finally, the pulse transit time changes with the cuff pressure changes (from 0 to $10 \mathrm{mmHg}$, from 10 to $20 \mathrm{mmHg}$, and from 20 to 30 $\mathrm{mmHg}$ ) were compared.Describe your methods here.

\section{Results}

Figure 2 shows one example of processed pulse transit time data when $30 \mathrm{mmHg}$ cuff pressure was applied to the lower arm, upper arm and whole arm, which compared with the normal condition without any external pressure. It shows clearly that the pulse transit times with the cuff on different positions are different.

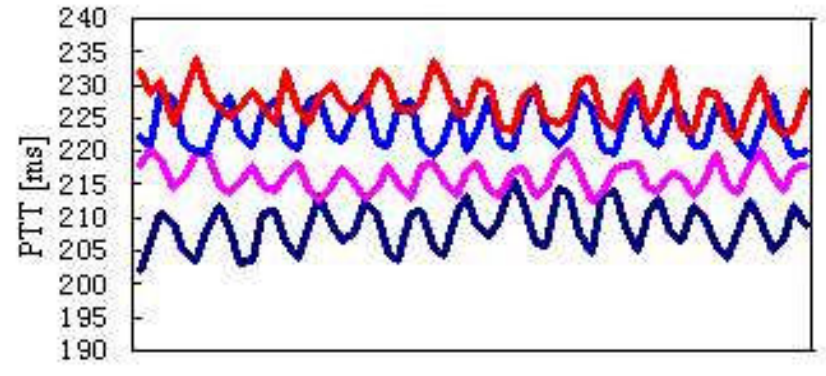

Figure 2: Example of pulse transit time when $30 \mathrm{mmHg}$ cuff pressure was applied. Form the top to the bottom, it shows the PTT with cuff pressure on the whole arm, upper arm, lower arm and no cuff pressure, respectively. The oscillations relate to respiratory cycles. 
Figure 3 shows overall mean and standard deviation of pulse transit time value from 60 consecutive beats for all 10 subjects. The overall mean pulse transit time with the above different cuff pressures on the lower arm were $219.5 \mathrm{~ms}, 222.8 \mathrm{~ms}, 226.4 \mathrm{~ms}$, and $229.5 \mathrm{~ms}$. With the cuff on the upper arm, the values were $219.8 \mathrm{~ms}, 223.5$ $\mathrm{ms}, 228.3 \mathrm{~ms}$ and $232.9 \mathrm{~ms}$. For the whole arm, they were $220.4 \mathrm{~ms}, \quad 225.9 \mathrm{~ms}, 231.9 \mathrm{~ms}$, and $238.4 \mathrm{~ms}$, respectively. Therefore, PTTs with the different cuff pressures are clearly distinguishable in this subject group.

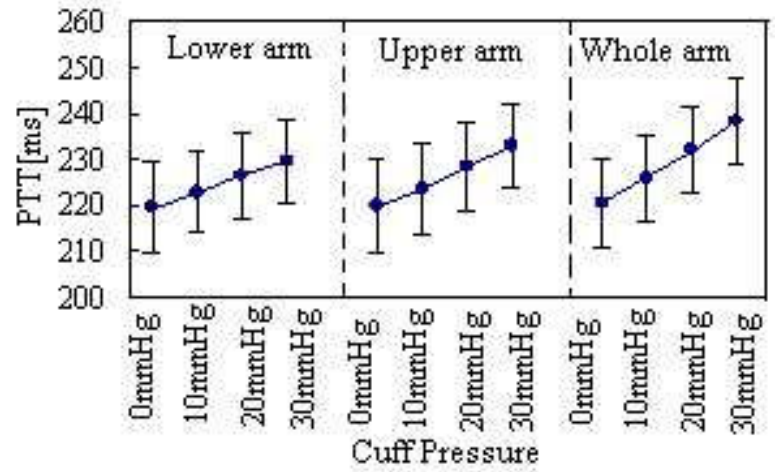

Figure 3: Overall mean and standard deviation of pulse transit time value with different cuff pressures. (Left part---cuff on the lower arm; middle part----cuff on the upper arm and right part---cuffs on the whole arm). Error bars relate mainly to subject differences. Whole arm means putting lower and upper cuffs simultaneously.

The changes of pulse transit time with different cuff pressures on different arms are shown in figure 4 . The overall mean pulse transit time difference with the above different cuff pressures on the lower arm compared with that without cuff pressure were $3.3 \mathrm{~ms}, 6.9 \mathrm{~ms}, 10.1 \mathrm{~ms}$. With the cuff on the upper arm, changes with increasing cuff pressure were greater, which were $3.7 \mathrm{~ms}, 8.5 \mathrm{~ms}$ and $13.1 \mathrm{~ms}$. And for the whole arm, the changes were $5.6 \mathrm{~ms}, 11.6 \mathrm{~ms}$ and $18.0 \mathrm{~ms}$. All these changes were significant $(\mathrm{P}<0.001)$.

Figure 5 shows the mean and standard deviation of the differences in pulse transit time under each different cuff pressure applied to the lower arm, upper arm and whole arm. Three comparisons were studied. With the cuff pressure of $10 \mathrm{mmHg}, 20 \mathrm{mmHg}$ and $30 \mathrm{mmHg}$, the mean pulse transit time when cuff pressure was applied to the whole arm was significantly larger $(\mathrm{P}<0.001)$ than the corresponding mean pulse transit time when cuff pressure was applied to the lower arm and upper arm. However, with cuff pressure of $10 \mathrm{mmHg}$, the mean pulse transit time with the cuff on the upper arm was not significantly larger than the corresponding mean pulse transit time with cuff on lower arm.

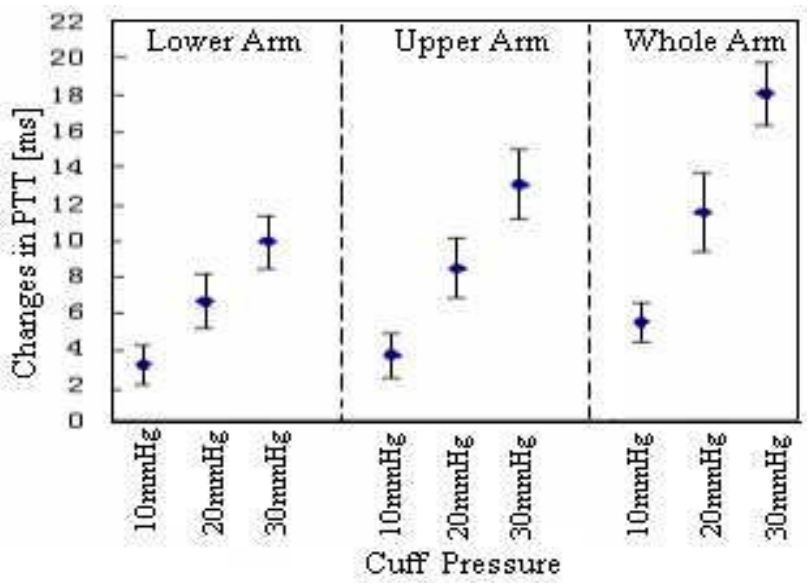

Figure 4: Overall mean and standard deviation of changes in pulse transit time with different cuff pressure on different arms. (Left part----cuff on the lower arm; middle part----cuff on the upper arm and right part-cuffs on the whole arm).
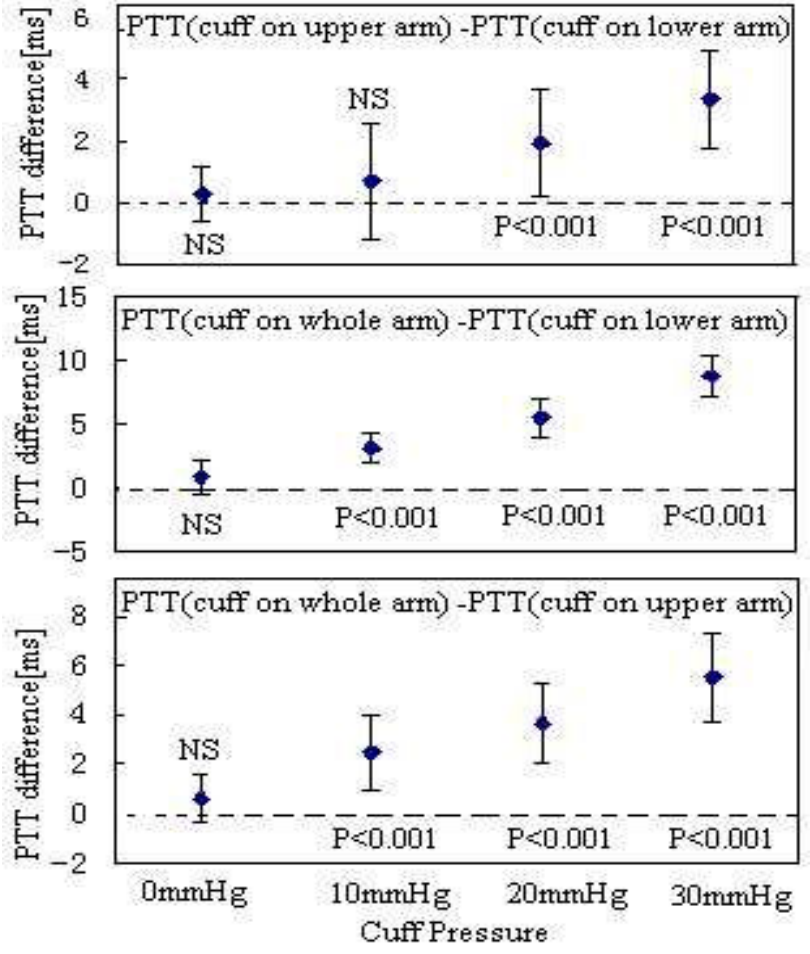

Figure 5: Comparisons of the differences in pulse transit time with each different cuff pressure on the lower arm, upper arm and whole arm. The mean and standard deviation of pulse transit time difference from each comparison are given.

Figure 6 shows the compared results of pulse transit time changes with the cuff pressure changing (from 0 to 10 $\mathrm{mmHg}$, from 10 to $20 \mathrm{mmHg}$, and from 20 to $30 \mathrm{mmHg}$ ). With the cuff pressure increasing by the same value, all 
the pulse transit time changes with the cuff on whole arm were significantly larger than those of lower and upper arm, which were not observed on the all the comparisons between the cuff on upper arm and the cuff on lower arm. In addition, the sum of pulse transit time changes with the cuff on the upper and lower arm was significantly larger than that with the cuff on whole arm.

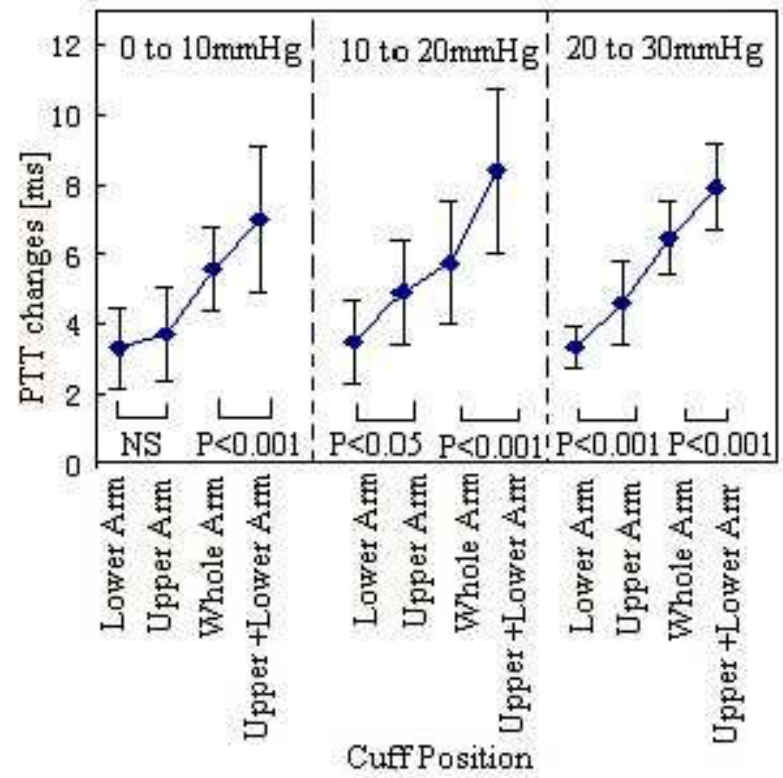

Figure 6: Comparisons of the pulse transit time changes with the cuff pressure changes (from 0 to $10 \mathrm{mmHg}$, from 10 to $20 \mathrm{mmHg}$, and from 20 to $30 \mathrm{mmHg}$ ) when cuff is on different positions. The pulse transit time changes of upper + lower arm were the sum of pulse transit time changes with the cuff on upper and lower arm.

\section{Discussion and conclusions}

The first finding about the changes of pulse transit time is when cuff pressure was increased from $0 \mathrm{mmHg}$ to 30 $\mathrm{mmHg}$, the mean pulse transit time value was significantly increased. The result agrees with the theoretical explanation. With a constant arterial pressure (blood pressure), when the external pressure increases, the arterial compliance increases, which leads to the increasing pulse transit time.

The second finding of this study is, under the same applied cuff pressure level, the mean pulse transit time when the cuff pressure was applied to the whole arm was significantly larger than the corresponding mean pulse transit time when the cuff pressure was applied to the lower arm and upper arm. The main reason is, with the cuff on whole arm, the size of artery with external pressure is twice that when the cuff is on upper or lower arm only. Also, with the cuff pressure of $20 \mathrm{mmHg}$ and
$30 \mathrm{mmHg}$, the mean pulse transit time with the cuff on upper arm was significantly larger than the corresponding mean pulse transit time when cuff is on lower arm, which may be caused by the different arterial structure characteristics and anatomy in the upper arm and in the lower arm.

The limitation of this study is the assumption of no reduction of applied cuff pressure through the skin, muscle and other tissues to the artery, however, in reality, it is impossible. So the effect of the reduction of cuff pressure should be considered.

\section{Acknowledgements}

We would like to acknowledge the support of an Overseas Research Studentship (Universities UK) and an International Research Studentship (Newcastle University) for this project.

\section{References}

[1] Panner BM, Avolio AP, Hoeks A, Mancia G, Takazawa K. Methods and devices for measuring arterial compliance in human. Am J Hypertens 2002; 15: 743-53.

[2] McVeigh GE. Pulse waveform analysis and arterial wall properties. Hypertension 2003; 41:1010-1011.

[3] McVeigh GE, Hamilton PK, Morgan DR. Evaluation of mechanical arterial properties: clinical, experimental and therapeutic aspects. Clin Sci 2002; 102: 51-67

[4] Drinnan MJ, Allen J, Murray A. Relation between heart rate and pulse transit time during paced respiration. Physiol Meas 2001; 22 425-31.

[5] Allen J, Murray A. Age-related changes in peripheral pulse timing characteristics at the ears, fingers and toes. J Human Hypertension 2002; 16: 711-7.

[6] Yamakoshi K, Shimazu H, Shibata M, Kamiya A. New oscillometric method for indirect measurement of systolic and mean arterial pressure in the human finger. Part I:Model experiment. Med Biol Eng Comput 1982; 20: $307-$ 13.

[7] Yamakoshi K, Shimazu H, Shibata M, Kamiya A. New oscillometric method for indirect measurement of systolic and mean arterial pressure in the human finger. Part II: correlation study. Med Biol Eng Comput 1982; 20: 314-8.

[8] Raamat R, Talts J, Jagomagi K, Lansimies E. Mathematical modelling of non-invasive oscillometric finger mean blood pressure measurement by maximum oscillation criterion. Med Biol Eng Comput 1999; 37: 784-8.

Address for correspondence

Name: Dingchang Zheng

Full postal address:

Medical Physics Department,

University of Newcastle upon Tyne, Freeman Hospital,

Newcastle upon Tyne NE7 7DN

E-mail address: Dingchang.zheng@ncl.ac.uk 